\title{
Dispersionless integrable systems and the Bogomolny equations on an Einstein-Weyl geometry background *
}

\author{
L.V. Bogdanov ${ }^{\dagger}$ \\ Landau Institute for Theoretical Physics, RAS, Moscow, Russia
}

\begin{abstract}
We derive a dispersionless integrable system describing a local form of a general three-dimensional Einstein-Weyl geometry with an Euclidean (positive) signature, construct its matrix extension and demonstrate that it leads to the Bogomolny equations for a non-abelian monopole on an Einstein-Weyl geometry background. The corresponding dispersionless integrable hierarchy, its matrix extension and the dressing scheme are also considered.
\end{abstract}

Keywords: Dispersionless integrable system, Einstein-Weyl geometry, Bogomolny equations, Yang-Mills-Higgs equations

\section{Introduction}

The present paper is a continuation of the work [1], where an integrable matrix extension of the Manakov-Santini system [2], [3] was introduced and it was demonstrated that it describes a $(2+1)$-dimensional integrable chiral model in an Einstein-Weyl space. Since the Manakov-Santini system corresponds to a local form of a general Lorentzian Einstein-Weyl geometry [4] (in the complex case, to a general complex analytic one), its integrable matrix extension gives a local description of a general case of the integrable chiral model model on an Einstein-Weyl geometry background.

The structure of the Bogomolny equations for a non-abelian monopole (see, e.g., [5]) is analogous to the Yang-Mills-Higgs equations, representing a reduction of the self-dual Yang-Mills equations and leading to the integrable chiral model [6], but, in contrast to it, they are written in Euclidean, not in Lorentzian three-dimensional space. To consider these equations on an integrable geometric

\footnotetext{
${ }^{*}$ This research was performed in the framework of State Assignment Topic 0033-2019-0006 (Integrable systems of Mathematical Physics).

†leonid@itp.ac.ru
} 
background, one needs a local description of an Einstein-Weyl geometry with a positive (Euclidean) signature.

It is problematic to perform a reduction to real case with Euclidean signature for the complex Manakov-Santini system because of asymmetry with respect to independent variables. A more suitable candidate is a local description of an Einstein-Weyl geometry using a generalisation of a dispersionless $(2+1)$ dimensional Toda equation (d2DTL) [7]

$$
\begin{aligned}
& \left(e^{-\phi}\right)_{t t}=m_{t} \phi_{x y}-m_{x} \phi_{t y}, \\
& m_{t t} e^{-\phi}=m_{t y} m_{x}-m_{x y} m_{t},
\end{aligned}
$$

with the Lax pair

$$
\begin{aligned}
\partial_{x} \boldsymbol{\Psi} & =\left(\left(\lambda+\frac{m_{x}}{m_{t}}\right) \partial_{t}-\left(\phi_{t} \frac{m_{x}}{m_{t}}-\phi_{x}\right) \lambda \partial_{\lambda}\right) \boldsymbol{\Psi}, \\
\partial_{y} \boldsymbol{\Psi} & =\left(-\frac{1}{\lambda} \frac{e^{-\phi}}{m_{t}} \partial_{t}-\frac{1}{\lambda} \frac{\left(e^{-\phi}\right)_{t}}{m_{t}} \lambda \partial_{\lambda}\right) \boldsymbol{\Psi} .
\end{aligned}
$$

For $m=t$ system (1) reduces to dispersionless (2+1)-dimensional Toda equation (d2DTL)

$$
\left(e^{-\phi}\right)_{t t}=\phi_{x y},
$$

A conformal structure of an Einstein-Weyl geometry is defined by the symbol of linearization of the equation representing a symmetric bivector (metric with upper indices) [8], for equation (3) it is

$$
h=\partial_{x} \partial_{y}+\mathrm{e}^{-\phi} \partial_{t}^{2}
$$

In this case it is easy to obtain a real metric with an Euclidean signature substituting $x=z, y=\bar{z}$, where $z=x^{1}+\mathrm{i} x^{2}$, to equation (3), which gives

$$
\left(e^{-\phi}\right)_{t t}=\phi_{z \bar{z}}
$$

and the corresponding conformal structure

$$
h=\partial_{z} \partial_{\bar{z}}+\mathrm{e}^{-\phi} \partial_{t}^{2}
$$

has an Euclidean signature in terms of real variables $x^{1}, x^{2}, t$. Lax pair for this equation can be written in a symmetric form

$$
\begin{array}{ll}
\partial_{z} \Psi=L_{1} \Psi, & L_{1}=\lambda \mathrm{e}^{\varphi} \partial_{t}-\left(\varphi_{z}+\lambda \mathrm{e}^{\varphi} \varphi_{\tau}\right) \lambda \partial_{\lambda}, \\
\partial_{\bar{z}} \boldsymbol{\Psi}=L_{2} \boldsymbol{\Psi}, & L_{2}=-\frac{1}{\lambda} \mathrm{e}^{\varphi} \partial_{t}+\left(\varphi_{\bar{z}}-\frac{1}{\lambda} \mathrm{e}^{\varphi} \varphi_{\tau}\right) \lambda \partial_{\lambda},
\end{array}
$$

here $\varphi=-\frac{1}{2} \phi$. For real $\varphi$ we have a symmetry $L_{2}(\lambda)=\bar{L}_{1}\left(-\bar{\lambda}^{-1}\right)$. Just this symmetry is responsible for real Euclidean reduction of the conformal structure, it is present for higher hierarchy operators also. 
For system (1) similar reduction does not work due to the asymmetry with respect to variables $x, y$. However, it was demonstrated in [7] that using a rather simple complex transformation involving a dependent variable $m$ and an independent variable $t$ it is possible to bring system (1) and its Lax pair to a symmetric form, providing real Euclidean reduction. This transformation looks natural in terms of the hierarchy connected with system (1).

The goal of the present work is to construct a symmetric analogue of equations (1) and their integrable matrix extension and a corresponding local form of an Einstein-Weyl geometry with an Euclidean signature. We will demonstrate that the matrix extension describes the Bogomolny equations on an EinsteinWeyl geometry background.

We will take system (1) as a basic system, corresponding Einstein-Weyl geometry was found in the work [8]. First we will demonstrate that matrix extension of this system, constructed in the work [1], gives the Yang-Mills-Higgs equations [5] on the Lorentzian Einstein-Weyl geometry background and, after fixing the gauge, an integrable chiral model (this conjecture was made but not proved in the work [1]). Then using a complex transformation we will proceed to a symmetric analogue of equations (1) and the Lax pair, construct their integrable matrix extension and demonstrate that it represents the Bogomolny equations on an Einstein-Weyl geometry background (the Yang=Mills-Higgs equations [5] on an Euclidean Einstein-Weyl geometry background). In the final part of the work we will build the hierarchy for symmetric analogue of equations (1) and its matrix extension and outline the dressing method scheme.

Twistor integrability of geometric structures related to our paper was established in the works [9], [10], [11]. Integrable background geometries for different reductions of the self-dual Yang-Mills equations connected with dispersionless integrable systems are considered in the paper [12]. The scheme of integrable matrix extension of dispersionless integrable systems was proposed in the work [13].

\section{A matrix extension of system (1)}

\subsection{An Einstein-Weyl geometry}

The Einstein-Weyl geometry corresponding to system (1) was found in the work [8]. A symbol of formal linearisation of system (1) leads to a symmetric bivector of a conformal structure

$$
h \sim e^{-\phi} \partial_{t}^{2}-m_{x} \partial_{y} \partial_{t}+m_{t} \partial_{x} \partial_{y},
$$

which corresponds to the metric

$$
g=\left(m_{x} d x+m_{t} d t\right)^{2}+4 e^{-\phi} m_{t} d x d y,
$$

satisfying together with a differential form

$$
\omega=\left(\frac{m_{t t}}{m_{t}^{2}}-2 \frac{\phi_{t}}{m_{t}}\right)\left(m_{x} d x+m_{t} d t\right)+2 \frac{m_{y t}}{m_{t}} d y
$$


Einstein-Weyl equations.

Let us remind (see [8], [14] for more detail), that a Weyl space is a manifold with a conformal structure $[g]$ and a symmetric connection $D$ consistent with $[g]$ in a sense that for every $g \in[g]$

$$
D g=\omega \otimes g
$$

for some differential form $\omega$ (the connection preserves a conformal class). The change of a metric representing a conformal structure $g \rightarrow f g$, where the function $f \neq 0$, corresponds to the transformation $\omega \rightarrow \omega+\mathrm{d} \ln f$. For the case of closed form $\omega$ it is possible locally to choose such a conformal gauge that the connection $D$ coincides with a metric connection. In a general case the form $\omega$ defines a difference of the connection $D$ from a metric Levi-Civita connection $\nabla$, explicit formulae for Christoffel symbols of the connection $D$ in terms of the form $\omega$ and Levi-Civita connection for a metric $g$ are given, e.g., in the work $[14]$

$$
D_{i} V^{j}=\nabla_{i} V^{j}+\gamma_{i k}^{j} V^{k},
$$

here

$$
\gamma_{i k}^{j}=-\frac{1}{2}\left(\delta_{i}^{j} \omega_{k}+\delta_{k}^{j} \omega_{i}-g_{i k} g^{j m} \omega_{m}\right) .
$$

Einstein-Weyl spaces are defined by the condition that the trace-free part of the symmetrised Ricci tensor of the connection $D$ vanishes (Einstein equations), which together with relation (9) constitute Einstein-Weyl equations system, in a coordinate form

$$
D_{i} g_{i j}=\omega_{i} g_{i j}, \quad R_{(i j)}=\Lambda g_{i j},
$$

here $\Lambda$ is some function. Einstein-Weyl equations are correctly defined for arbitrary manifold dimension not less than three, but the most interesting case is three-dimensional when they are integrable [11]. We cosider only threedimensional Einstein-Weyl geometry in the present work.

\subsection{A matrix extension and the Yang-Mills-Higgs equa- tions}

In the work [1] we introduced a matrix extension of Lax pair (2)

$$
\begin{aligned}
& L_{1}=\partial_{x}-\left(\lambda+\frac{m_{x}}{m_{t}}\right) \partial_{t}+\lambda\left(\phi_{t} \frac{m_{x}}{m_{t}}-\phi_{x}\right) \partial_{\lambda}+A \\
& L_{2}=\partial_{y}+\frac{1}{\lambda} \frac{\mathrm{e}^{-\phi}}{m_{t}} \partial_{t}+\frac{\left(\mathrm{e}^{-\phi}\right)}{m_{t}} \partial_{\lambda}+\frac{1}{\lambda} B,
\end{aligned}
$$

where $A, B$ are matrices (Lie algebra elements). Commutativity conditions for the matrix extension lead simultaneously to system (1) and matrix equations 
on the background of solutions of this system

$$
\begin{aligned}
& \partial_{t} B+\partial_{y} A=0, \\
& \left(\partial_{x}-\frac{m_{x}}{m_{t}} \partial_{t}\right) B-\frac{\mathrm{e}^{-\phi}}{m_{t}} \partial_{t} A-\left(\phi_{t} \frac{m_{x}}{m_{t}}-\phi_{x}\right) B+[A, B]=0,
\end{aligned}
$$

or, in terms of potential $K, A=K_{t}, B=-K_{y}$,

$$
\mathrm{e}^{-\phi} K_{t t}-m_{x} K_{y t}+m_{t} K_{x y}-\left(\phi_{t} m_{x}-\phi_{x} m_{t}\right) K_{y}-m_{t}\left[K_{y}, K_{t}\right]=0 .
$$

This equation on the trivial background $m=t, \phi=1$ coincides (up to a change of variables) with an integrable chiral model [5].

We would like to demonstrate that the matrix extension of system (1) corresponds to the Yang-Mills-Higgs equations on an Einstein-Weyl geometry (7), (8) background, similar to the result obtained in the work [1] for the matrix extension of the Manakov-Santini system. The Yang-Mills-Higgs equations on an Einstein-Weyl geometry background read

$$
\mathrm{D} \Phi+\frac{1}{2} \omega \Phi=* F
$$

where a 2-form $F=\mathrm{d} A+A \wedge A$ ( curvature of the connection, gauge field intensity), $A$ is a gauge field (potential) representing a 1 -form with the values in some (matrix) Lie algebra, $\Phi$ is a function taking values in the Lie algebra (Higgs field, $[5]), \mathrm{D} \Phi=\mathrm{d} \Phi+[A, \Phi]$. The form $\omega$ together with the metric $g$ satisfy Einstein-Weyl equations (10). For the Minkowski metric equation (14) coincides with the Yang-Mills-Higgs system introduced by Ward [6], leading to an integrable chiral model, in the Euclidean case it gives the Bogomolny equations (see, e.g., [5]). Equation (14) is invariant under the change of a conformal gauge of the Einstein-Weyl geometry $g \rightarrow f g, \omega \rightarrow \omega+\mathrm{d} \ln f, \Phi \rightarrow$ $f-\frac{1}{2} \Phi$.

We will introduce a special basis of vector fields and a dual basis of differential forms in which metric (7) takes a simple form, and will consider components of equation (14) in this basis with the use of a standard formula

$$
F(u, v)=\nabla_{u} \nabla_{v}-\nabla_{v} \nabla_{u}-\nabla_{[u, v]}
$$

which is valid for arbitrary vector fields $u, v$.

Let us introduce a basis of vector fields

$$
\mathbf{e}_{1}=\partial_{x}-\frac{m_{x}}{m_{t}} \partial_{t}, \quad \mathbf{e}_{2}=\partial_{y}, \quad \mathbf{e}_{3}=\partial_{t},
$$

in which symmetric bivector of conformal structure (6) is represented as

$$
h=\mathrm{e}^{\phi} m_{t} \mathbf{e}_{1} \cdot \mathbf{e}_{2}+\left(\mathbf{e}_{3}\right)^{2} .
$$

The dual basis of 1 -forms reads

$$
\mathbf{e}^{1}=\mathrm{d} x, \quad \mathbf{e}^{2}=\mathrm{d} y, \quad \mathbf{e}^{3}=\left(\mathrm{d} t+\frac{m_{x}}{m_{t}} \mathrm{~d} x\right),
$$


and for metric (7) we obtain

$$
g=4 \frac{\mathrm{e}^{-\phi}}{m_{t}} \mathbf{e}^{1} \cdot \mathbf{e}^{2}+\left(\mathbf{e}_{3}\right)^{2}
$$

(up to a conformal factor $m_{t}^{-2}$ ).

Let us consider gauge covariant vector fields $\nabla_{i}=\nabla_{\mathbf{e}_{i}}=\mathbf{e}_{i}+A_{i}$. In terms of these fields Lax pair (11) takes the form

$$
\begin{aligned}
L_{1} & =\nabla_{1}-\lambda\left(\nabla_{3}+\Phi\right)+\left(\phi_{t} \frac{m_{x}}{m_{t}}-\phi_{x}\right) \lambda \partial_{\lambda}, \\
L_{2} & =\nabla_{2}+\frac{1}{\lambda} \frac{\mathrm{e}^{-\phi}}{m_{t}}\left(\nabla_{3}-\Phi\right)+\frac{1}{\lambda} \frac{\left(\mathrm{e}^{-\phi}\right)_{t}}{m_{t}} \lambda \partial_{\lambda}
\end{aligned}
$$

Operators (11) correspond to a special gauge compatible with commutativity conditions. We will consider matrix extension (20) without fixing a gauge. From the commutativity equations we obtain

$$
\begin{aligned}
& -\frac{1}{2} \mathrm{e}^{\phi} m_{t}\left[\nabla_{1}, \nabla_{2}\right]=\left[\nabla_{3}, \Phi\right]+\frac{1}{2} \frac{m_{t t}}{m_{t}} \nabla_{3}-\frac{1}{2}\left(2 \phi_{t}+\frac{m_{t t}}{m_{t}}\right) \Phi, \\
& {\left[\nabla_{2}, \nabla_{3}+\Phi\right]=0,} \\
& {\left[\nabla_{1}, \nabla_{3}-\Phi\right]=\left(\frac{m_{x}}{m_{t}}\right)_{t}\left(\nabla_{3}-\Phi\right)}
\end{aligned}
$$

We have omitted commutativity equations corresponding to the terms containing a derivative over spectral variable $\partial_{\lambda}$. These equations do not contain a matrix part and they reduce to the first equation of system (1). The second equation of this system arises from the scalar (corresponding to commutation of vector fields) part of relation (21). Relation (22) is responsible for the existence of a gauge in which system (20) reduces to a form (11).

According to formula (15), using commutativity equations, for metric (19) in the basis (18) we obtain

$$
\begin{aligned}
& (* F)_{3}=-\frac{1}{2} \mathrm{e}^{\phi} m_{t}\left[\nabla_{1}, \nabla_{2}\right]-\frac{1}{2} \frac{m_{t t}}{m_{t}} \nabla_{3}, \\
& (* F)_{2}=\left[\nabla_{3}, \nabla_{2}\right], \\
& (* F)_{1}=\left[\nabla_{1}, \nabla_{3}\right]-\left(\frac{m_{x}}{m_{t}}\right)_{t} \nabla_{3} .
\end{aligned}
$$

A vector field in the r.h.s. of the first expression cancels due to the second equation of system (1), in the second it is absent, in the third it cancels identically. Comparing the matrix part of compatibility equations (21-23) with Yang-MillsHiggs equations (14), we come to the conclusion that it represents equations (14) with a 1 -form

$$
\begin{aligned}
& \omega=-2\left(\frac{m_{x}}{m_{t}}\right)_{t} \mathbf{e}^{1}-\left(2 \phi_{t}+\frac{m_{t t}}{m_{t}}\right) \mathbf{e}^{3} \\
& =\left(\frac{m_{t t}}{m_{t}} \frac{m_{x}}{m_{t}}-2 \phi_{t} \frac{m_{x}}{m_{t}}-2 \frac{m_{x t}}{m_{t}}\right) \mathrm{d} x-\left(2 \phi_{t}+\frac{m_{t t}}{m_{t}}\right) \mathrm{d} t,
\end{aligned}
$$


which, up to a conformal gauge corresponding to metric (19), $\omega \rightarrow \omega+2 \mathrm{~d} \ln m_{t}$, coincides with 1 -form (8).

Proposition 1. The matrix part of commutativity equations for Lax pair (20) represents Yang-Mills-Higgs equations (14) on the background of Einstein-Weyl geometry (7-8)

Using the statements of the work [4] that Einstein-Weyl geometry (7-8) connected with system (1) is a local form of a generic Lorentzian (or, in the complex analytic case, generic complex analytic) three-dimensional EinsteinWeyl geometry, we come to the following conclusion:

Proposition 2. There exist local coordinates such that Yang-Mills-Higgs equations (14) on the background of real Lorentzian (general complex analytic) EinsteinWeyl geometry reduce to equations of commutativity of operators (20), so that the scalar part of commutativity conditions corresponds to system (1) defining an Einstein-Weyl geometry (7), (8), and the matrix (gauge) part gives the YangMills-Higgs equations on the background of this Einstein-Weyl geometry.

\section{The case of an Einstein-Weyl geometry with the Euclidean signature}

\subsection{A symmetric Lax pair and equations}

The technique needed to make a transition from system (1) to the system symmetric in variables $x, y$, describing an Einstein-Weyl geometry with the Euclidean signature, was developed in the work [7]. This transition is described by a rather simple transformation

$$
\begin{gathered}
t=\tau+\mathrm{i} \mu, \\
m=\tau-\mathrm{i} \mu,
\end{gathered}
$$

where $\tau$ is a new independent variable, and $\mu$ is a new dependent variable. This transformation is considered for Einstein-Weyl geometry (1), (7), (8) in the complex analytic case, and a real Euclidean reduction is defined by the condition that $\phi, \mu, \tau$ are real and $x, y$ are complex conjugated, $x=z, y=\bar{z}$. To perform a transition it is convenient to rewrite system (1) in terms of differential forms,

$$
\begin{aligned}
& \mathrm{d}\left(\mathrm{e}^{-\phi}\right)_{t} \wedge \mathrm{d} x \wedge \mathrm{d} y=\mathrm{d} m \wedge d \phi_{y} \wedge \mathrm{d} y \\
& \mathrm{e}^{-\phi} \mathrm{d} m_{t} \wedge \mathrm{d} x \wedge \mathrm{d} y=-\mathrm{d} m \wedge \mathrm{d} m_{y} \wedge \mathrm{d} y
\end{aligned}
$$

Having in mind the matrix extension and the Yang-Mills-Higgs equations, we will perform the transformation on the level of a Lax pair.

The transformation to a symmetric system arises naturally if one considers the symmetries of linear operators and wave functions of the hierarchy, analogous to the symmetry of Lax pair (5) for a simpler case of equation (3). We will 
give a brief description in terms of the hierarchy below, and now we will move on to a symmetric analogue of Lax pair (2) and construct its matrix extension, similar to matrix extension (20) for Lax pair (2).

A symmetric Lax pair

$$
\begin{array}{ll}
\partial_{z} \Psi=L_{1} \Psi, & L_{1}=\left(\lambda \mathrm{e}^{\varphi} u+v\right) \partial_{\tau}+\left(\left(\varphi_{\tau} v-\varphi_{z}\right)-\lambda u \mathrm{e}^{\varphi} \varphi_{\tau}\right) \lambda \partial_{\lambda}, \\
\partial_{\bar{z}} \boldsymbol{\Psi}=L_{2} \boldsymbol{\Psi}, & L_{2}=\left(-\frac{1}{\lambda} \mathrm{e}^{\varphi} \bar{u}+\bar{v}\right) \partial_{\tau}-\left(\left(\varphi_{\tau} \bar{v}-\varphi_{\bar{z}}\right)+\frac{1}{\lambda} \overline{\mathrm{e}} \mathrm{e}^{\varphi} \varphi_{\tau}\right) \lambda \partial_{\lambda}
\end{array}
$$

where $\varphi=-\frac{1}{2} \phi$, is obtained from Lax pair (2) by a transformation (26) plus an extra twisting of spectral variable $\lambda \rightarrow \mathrm{e}^{\varphi} \lambda$, it possesses a symmetry $L_{2}(\lambda)=$ $\bar{L}_{1}\left(-\bar{\lambda}^{-1}\right)$. Here we have introduced the notations

$$
u=\frac{1}{1+\mathrm{i} \mu_{\tau}}, \quad \bar{u}=\frac{1}{1-\mathrm{i} \mu_{\tau}}, \quad \bar{v}=\frac{\mathrm{i} \mu_{\bar{z}}}{1+\mathrm{i} \mu_{\tau}}, \quad v=-\frac{\mathrm{i} \mu_{z}}{1-\mathrm{i} \mu_{\tau}} .
$$

Equations of commutativity of the Lax pair read

$$
\begin{aligned}
& \left(v_{\bar{z}}-\mathrm{e}^{\varphi} u \partial_{\tau}\left(\mathrm{e}^{\varphi} \bar{u}\right)+v \partial_{\tau} \bar{v}\right)-\text { c.c. }=0, \\
& \left(\partial_{\bar{z}}\left(\varphi_{\tau} v-\varphi_{z}\right)-\mathrm{e}^{\varphi} u \partial_{\tau}\left(\bar{u} \mathrm{e}^{\varphi} \varphi_{\tau}\right)-v \partial_{\tau}\left(\varphi_{\tau} \bar{v}-\varphi_{\bar{z}}\right)-u \bar{u} \mathrm{e}^{2 \varphi} \varphi_{\tau} \varphi_{\tau}\right) \\
& \quad+\text { c.c. }=0
\end{aligned}
$$

or, in explicit form,

$$
\begin{aligned}
& \frac{\mathrm{e}^{2 \varphi}}{1+\mu_{\tau}^{2}} \mu_{\tau \tau}+\mu_{z \bar{z}}+\frac{\mu_{z} \mu_{\bar{z}} \mu_{\tau \tau}}{1+\mu_{\tau}^{2}}-\frac{\mathrm{i} \mu_{\bar{z}} \mu_{\tau z}}{1+\mathrm{i} \mu_{\tau}}+\frac{\mathrm{i} \mu_{z} \mu_{\tau \bar{z}}}{1-\mathrm{i} \mu_{\tau}}=0 \\
& \frac{1}{2} \frac{\left(\mathrm{e}^{2 \varphi}\right)_{\tau \tau}}{1+\mu_{\tau}^{2}}+\varphi_{z \bar{z}}+\frac{\mu_{z} \mu_{\bar{z}} \varphi_{\tau \tau}}{1+\mu_{\tau}^{2}}+\frac{\mathrm{i} \mu_{z} \varphi_{\tau \bar{z}}}{1-\mathrm{i} \mu_{\tau}}-\frac{\mathrm{i} \mu_{\bar{z}} \varphi_{\tau z}}{1+\mathrm{i} \mu_{\tau}}+F \varphi_{\tau}=0 \\
& 2 F=\left(\frac{\mu_{z} \mu_{\bar{z}}}{1+\mu_{\tau}^{2}}\right)_{\tau}+\left(\frac{\mathrm{i} \mu_{z}}{1-\mathrm{i} \mu_{\tau}}\right)_{\bar{z}}-\left(\frac{\mathrm{i} \mu_{\bar{z}}}{1+\mathrm{i} \mu_{\tau}}\right)_{z} .
\end{aligned}
$$

These equations can be obtained directly from system (1) by transformation (26), also giving the corresponding Einstein-Weyl structure, which we will write down below considering the matrix extension.

\section{Reductions}

Let us consider characteristic reductions of system (28-29).

A Hamiltonian reduction for vector fields of Lax pair (27) corresponds to $\mu=$ $0(u=1, v=0)$, equation (28) vanishes, equation (29) reduces to dispersionless $(2+1)$-dimensional Toda equation $(3)$ for $\phi=(-2 \varphi)$.

A linearly degenerate case for which vector fields of the Lax pair do not contain a derivative over the spectral variable corresponds to $\varphi=0$, equation (29) vanishes, from equation (28) we get

$$
\left(1+\mu_{z} \mu_{\bar{z}}\right) \mu_{\tau \tau}+\left(1+\mu_{\tau}^{2}\right) \mu_{z \bar{z}}-\mathrm{i}\left(1-\mathrm{i} \mu_{\tau}\right) \mu_{\bar{z}} \mu_{\tau z}+\mathrm{i}\left(1+\mathrm{i} \mu_{\tau}\right) \mu_{z} \mu_{\tau \bar{z}}=0
$$


An interpolating reduction, which for system (1) looks like $\phi=\beta \ln m_{t}$ [7], [15], for system (28-29) takes the form

$$
\varphi=\beta \tan ^{-1} \mu_{\tau}
$$

and leads to the equation

$\left(\mathrm{e}^{\beta \tan ^{-1} \mu_{\tau}}+\mu_{z} \mu_{\bar{z}}\right) \mu_{\tau \tau}+\left(1+\mu_{\tau}^{2}\right) \mu_{z \bar{z}}-\mathrm{i}\left(1-\mathrm{i} \mu_{\tau}\right) \mu_{\bar{z}} \mu_{\tau z}+\mathrm{i}\left(1+\mathrm{i} \mu_{\tau}\right) \mu_{z} \mu_{\tau \bar{z}}=0$

The limit $\beta \rightarrow 0$ corresponds to the linearly-degenerate case (30), and $\beta \rightarrow \infty$ to the Hamiltonian reduction (3).

\subsection{A matrix extension}

Let us consider the matrix extension of Lax pair (27)

$$
\begin{aligned}
& L_{1}=\partial_{z}-\left(\lambda \mathrm{e}^{\varphi} u+v\right) \partial_{\tau}-\left(\left(\varphi_{\tau} v-\varphi_{z}\right)-\lambda u \mathrm{e}^{\varphi} \varphi_{\tau}\right) \lambda \partial_{\lambda}+A-\lambda B \\
& L_{2}=\partial_{\bar{z}}-\left(-\frac{1}{\lambda} \mathrm{e}^{\varphi} \bar{u}+\bar{v}\right) \partial_{\tau}+\left(\left(\varphi_{\tau} \bar{v}-\varphi_{\bar{z}}\right)+\frac{1}{\lambda} \bar{u} \mathrm{e}^{\varphi} \varphi_{\tau}\right) \lambda \partial_{\lambda}+\widetilde{A}+\lambda^{-1} \widetilde{B}
\end{aligned}
$$

where $A, B, \widetilde{A}, \widetilde{B}$ are some matrix-valued functions. We introduce a basis of vector fields

$$
\mathbf{e}_{1}=\partial_{z}-v \partial_{\tau}, \quad \mathbf{e}_{2}=\partial_{\bar{z}}-\bar{v} \partial_{\tau}, \quad \mathbf{e}_{3}=\partial_{\tau}
$$

and a dual basis of 1-forms

$$
\mathbf{e}^{1}=\mathrm{d} z, \quad \mathbf{e}^{2}=\mathrm{d} \bar{z}, \quad \mathbf{e}^{3}=(\mathrm{d} \tau+v \mathrm{~d} z+\bar{v} \mathrm{~d} \bar{z}) .
$$

Let us rewrite the matrix extension of the Lax pair in terms of gauge covariant vector fields $\nabla_{i}=\nabla_{\mathbf{e}_{i}}=\mathbf{e}_{i}+A_{i}$ in the form

$$
\begin{aligned}
& L_{1}=\nabla_{1}-\lambda u \mathrm{e}^{\varphi}\left(\nabla_{3}-\mathrm{i} \Phi\right)-\left(\left(\varphi_{\tau} v-\varphi_{z}\right)-\lambda u \mathrm{e}^{\varphi} \varphi_{\tau}\right) \lambda \partial_{\lambda}, \\
& L_{2}=\nabla_{2}+\frac{1}{\lambda} \bar{u} \mathrm{e}^{\varphi}\left(\nabla_{3}+\mathrm{i} \Phi\right)+\left(\left(\varphi_{\tau} \bar{v}-\varphi_{\bar{z}}\right)+\frac{1}{\lambda} \bar{u} \mathrm{e}^{\varphi} \varphi_{\tau}\right) \lambda \partial_{\lambda}
\end{aligned}
$$

First consider the trivial background case, $\varphi=0, \mu=0$,

$$
\begin{aligned}
L_{1} & =\nabla_{z}-\lambda\left(\nabla_{\tau}-\mathrm{i} \Phi\right), \\
L_{2} & =\nabla_{\bar{z}}+\frac{1}{\lambda}\left(\nabla_{\tau}+\mathrm{i} \Phi\right)
\end{aligned}
$$

The conditions of commutativity of these operators read

$$
\begin{aligned}
& {\left[\nabla_{z}, \nabla_{\bar{z}}\right]=2 \mathrm{i}\left[\nabla_{\tau}, \Phi\right],} \\
& {\left[\nabla_{z}, \nabla_{\tau}\right]=-\mathrm{i}\left[\nabla_{z}, \Phi\right],} \\
& {\left[\nabla_{\bar{z}}, \nabla_{\tau}\right]=\mathrm{i}\left[\nabla_{\bar{z}}, \Phi\right],}
\end{aligned}
$$


or, in terms of real coordinates $x^{1}, x^{2}, \tau, z:=x^{1}+\mathrm{i} x^{2}$,

$$
\begin{aligned}
& {\left[\nabla_{x^{1}}, \nabla_{x^{2}}\right]=4\left[\nabla_{\tau}, \Phi\right],} \\
& {\left[\nabla_{x^{1}}, \nabla_{\tau}\right]=-\left[\nabla_{x^{2}}, \Phi\right],} \\
& {\left[\nabla_{x^{2}}, \nabla_{\tau}\right]=\left[\nabla_{x^{1}}, \Phi\right] .}
\end{aligned}
$$

It is easy to see that equations (34) represent the Bogomolny equations

$$
\mathrm{D} \Phi=* F
$$

for the Euclidean metric $g=4\left(\left(\mathrm{~d} x^{1}\right)^{2}+\left(\mathrm{d} x^{2}\right)^{2}\right)+(\mathrm{d} \tau)^{2}$, and equations (33) give a complex form of these equations with the expression for the metric $g=$ $4 \mathrm{~d} z \mathrm{~d} \bar{z}+(\mathrm{d} \tau)^{2}$.

Remark. Considering an imaginary coordinate $\tau=\mathrm{it}$ leads to Minkowski metric, and equations (33) in a special gauge give a symmetric version of the chiral model studied in [16].

Let us proceed to the general case. From the conditions of commutativity $\left[L_{1}, L_{2}\right]=0$ for operators (32) we get

$$
\begin{aligned}
& \frac{1}{2 \mathrm{i} u \bar{u} \mathrm{e}^{2 \varphi}}\left[\nabla_{1}, \nabla_{2}\right]=\left[\nabla_{3}, \Phi\right]+2 \varphi_{\tau} \Phi+\frac{1}{2 \mathrm{i}}\left(\frac{\bar{u}_{\tau}}{\bar{u}}-\frac{u_{\tau}}{u}\right) \nabla_{3}+\frac{1}{2}\left(\frac{\bar{u}_{\tau}}{\bar{u}}+\frac{u_{\tau}}{u}\right) \Phi \\
& {\left[\nabla_{1}, \nabla_{3}+\mathrm{i} \Phi\right]+\frac{\bar{u}_{z}-v \bar{u}_{\tau}}{\bar{u}}\left(\nabla_{3}+\mathrm{i} \Phi\right)=0} \\
& {\left[\nabla_{2}, \nabla_{3}-\mathrm{i} \Phi\right]+\frac{u_{\bar{z}}-\bar{v} u_{\tau}}{u}\left(\nabla_{3}-\mathrm{i} \Phi\right)=0,}
\end{aligned}
$$

a complete system of commutativity equation is obtained after adding equation (29), which corresponds to vanishing of the term with the derivative over a spectral variable $\partial_{\lambda}$.

A symmetric bivector of conformal structure can be obtained from formula (17) or from the symbol of linearisation of system (28-29),

$$
h=\left(1+\mu_{\tau}^{2}\right) \mathrm{e}^{-2 \varphi} \mathbf{e}_{1} \cdot \mathbf{e}_{2}+\left(\mathbf{e}_{3}\right)^{2},
$$

the metric reads, respectively,

$$
g=4\left(1+\mu_{\tau}^{2}\right)^{-1} \mathrm{e}^{2 \varphi} \mathbf{e}^{1} \cdot \mathbf{e}^{2}+\mathbf{e}^{3} \cdot \mathbf{e}^{3},
$$

its signature is Euclidean. Similar to the case of operators (31) it is possible to demonstrate that the matrix part of commutativity conditions (35) represents Yang-Mills-Higgs system (14)

$$
\mathrm{D} \Phi+\frac{1}{2} \omega \Phi=* F
$$

for metric (conformal structure) (37) and 1-form $\omega$

$$
\omega=-\left(\frac{\mathrm{i} \mu_{\bar{z}}}{1+\mathrm{i} \mu_{\tau}}\right)_{\tau} \mathbf{e}^{1}+\left(\frac{\mathrm{i} \mu_{z}}{1-\mathrm{i} \mu_{\tau}}\right)_{\tau} \mathbf{e}^{2}+\left(4 \varphi_{\tau}-\partial_{\tau} \ln \left(1+\mu_{\tau}^{2}\right)\right) \mathbf{e}^{3},
$$


or the Bogomolny equations on the background of an Einstein-Weyl geometry.

Results of the work [4] imply that real Einstein-Weyl geometry (37), (39) connected with system (28-29) gives a local form of a generic Einstein-Weyl geometry with Euclidean signature, and we come to the following conclusion:

Proposition 3. The Bogomolny equations on the background of an EinsteinWeyl geometry (real, with the Euclidean signature) (38) locally reduce to equations of commutativity of operators (32), so that the scalar part of commutativity conditions (connected with vector fields) corresponds to the system (28-29), defining an Einstein-Weyl geometry (37), (39), and the matrix (gauge) part gives the Bogomolny equations on the background of this Einstein-Weyl geometry.

\section{Hierarchies, a matrix extension, the dressing method}

In this part we will give a brief description of the hierarchies connected with systems (1) and (28-29) and their matrix extensions. Our presentation is based on the works [7], [15]. Considering the hierarchies clarifies the connections between the systems and the origin of transformation (26).

\subsection{The generalised dispersionless d2DTL hierarchy and its matrix extension}

The set of Lax-Sato equations of the hierarchy connected with system (1) reads [7], [15]

$$
\begin{aligned}
& \left(\partial_{x_{n}}-\left(\frac{\mathrm{e}^{n \Lambda} \lambda \partial_{\lambda} \Lambda}{\{\Lambda, M\}}\right)_{+}^{\text {out }} \partial_{t}+\left(\frac{\mathrm{e}^{n \Lambda} \partial_{t} \Lambda}{\{\Lambda, M\}}\right)_{+}^{\text {out }} \lambda \partial_{\lambda}\right)\left(\begin{array}{c}
\Lambda \\
M
\end{array}\right)=0 \\
& \left(\partial_{y_{n}}+\left(\frac{\mathrm{e}^{-n \Lambda} \lambda \partial_{\lambda} \Lambda}{\{\Lambda, M\}}\right)_{-}^{\text {in }} \partial_{t}-\left(\frac{\mathrm{e}^{-n \Lambda} \partial_{t} \Lambda}{\{\Lambda, M\}}\right)_{-}^{\text {in }} \lambda \partial_{\lambda}\right)\left(\begin{array}{c}
\Lambda \\
M
\end{array}\right)=0
\end{aligned}
$$

where a Poisson bracket is defined as $\{f, g\}=\lambda\left(f_{\lambda} g_{t}-f_{t} g_{\lambda}\right)$, and we consider formal series

$$
\begin{aligned}
& \Lambda^{\text {out }}=\ln \lambda+\sum_{k=1}^{\infty} l_{k}^{+} \lambda^{-k}, \quad \Lambda^{\text {in }}=\ln \lambda+\phi+\sum_{k=1}^{\infty} l_{k}^{-} \lambda^{k} \\
& M^{\text {out }}=M_{0}^{\text {out }}+\sum_{k=1}^{\infty} m_{k}^{+} \mathrm{e}^{-k \Lambda^{\text {out }}}, \quad M^{\text {in }}=M_{0}^{\text {in }}+m_{0}+\sum_{k=1}^{\infty} m_{k}^{-} \mathrm{e}^{k \Lambda^{\text {in }}}, \\
& M_{0}=t+\sum_{k=1}^{\infty} x_{k} \mathrm{e}^{k \Lambda}-\sum_{k=1}^{\infty} y_{k} \mathrm{e}^{-k \Lambda},
\end{aligned}
$$

where $\lambda$ is a spectral variable, $(\ldots)_{-},(\ldots)_{+}$are standard projectors of Laurent series to negative and non-positive powers respectively. Usually we suggest that 
'out' and 'in' components define the functions inside and outside the unit circle in the complex plane, with $\Lambda^{\text {in }}-\ln \lambda, M^{\text {in }}-M_{0}^{\text {in }}$ analytic in the unit disc and $\Lambda^{\text {out }}-\ln \lambda, M^{\text {out }}-M_{0}^{\text {out }}$ analytic outside the unit disc and decreasing at infinity. For a function on the complex plane, having a discontinuity on the unit circle, by 'in' and 'out' components we mean the function inside and outside the unit disc. Lax-Sato equations are equivalent to the generating relation

$$
\left(\{\Lambda, M\}^{-1} \mathrm{~d} \Lambda \wedge \mathrm{d} M\right)^{\text {out }}=\left(\{\Lambda, M\}^{-1} \mathrm{~d} \Lambda \wedge \mathrm{d} M\right)^{\mathrm{in}},
$$

suggesting analyticity of the 2-form

$$
\omega=\{\Lambda, M\}^{-1} \mathrm{~d} \Lambda \wedge \mathrm{d} M
$$

in the complex plane. The dressing scheme for hierarchy (41) can be formulated in terms of two-component nonlinear Riemann-Hilbert problem on the unit circle

$$
\begin{aligned}
& \Lambda^{\text {out }}=F_{1}\left(\Lambda^{\text {in }}, M^{\text {in }}\right), \\
& M^{\text {out }}=F_{2}\left(\Lambda^{\text {in }}, M^{\text {in }}\right),
\end{aligned}
$$

Lax-Sato equations for the times $x=x_{1}, y=y_{1}$ correspond to Lax pair (2).

To obtain a matrix extension of the hierarchy, we introduce in addition a matrix Riemann-Hilbert problem

$$
\Psi^{\text {out }}=\Psi^{\text {in }} R\left(\Lambda^{\text {in }}, M^{\text {in }}\right),
$$

the matrix-valued function $\Psi$ is normalised by 1 at infinity and analytic, having no zeroes, inside and outside the unit circle,

$$
\Psi^{\text {out }}=1+\sum_{n=1}^{\infty} \Psi_{n}^{+}(\mathbf{t}) \lambda^{-n}, \quad \Psi^{\text {in }}=\sum_{n=0}^{\infty} \Psi_{n}^{-}(\mathbf{t}) \lambda^{n} .
$$

Unity normalisation of the function $\Psi$ accounts for fixing the gauge corresponding to the operators of the form (11). General operators of matrix (gauge) extension of the type (20) require unnormalised regular (bounded at infinity) solutions of problem (46). Matrix Riemann problem implies analyticity of the matrix-valued 3-form

$$
\Omega=\omega \wedge \mathrm{d} \Psi \cdot \Psi^{-1},
$$

corresponding to an additional generating relation for the matrix extension of the hierarchy,

$$
\left(\omega \wedge \mathrm{d} \Psi \cdot \Psi^{-1}\right)^{\text {in }}=\left(\omega \wedge \mathrm{d} \Psi \cdot \Psi^{-1}\right)^{\text {out }},
$$

which gives Lax-Sato equations for series (47), defining their evolution on the background produced by Lax-Sato equations (41),

$$
\begin{aligned}
& \partial_{x_{n}}\left(\begin{array}{c}
\Lambda \\
M
\end{array}\right)=V_{n}^{+}(\lambda)\left(\begin{array}{c}
\Lambda \\
M
\end{array}\right), \quad \partial_{y_{n}}\left(\begin{array}{c}
\Lambda \\
M
\end{array}\right)=V_{n}^{-}(\lambda)\left(\begin{array}{c}
\Lambda \\
M
\end{array}\right), \\
& \partial_{x_{n}} \Psi=\left(V_{n}^{+}(\lambda)-\left(\left(V_{n}^{+}(\lambda) \Psi\right) \cdot \Psi^{-1}\right)_{+}^{\text {out }}\right) \Psi, \\
& \partial_{y_{n}} \Psi=\left(V_{n}^{-}(\lambda)-\left(\left(V_{n}^{-}(\lambda) \Psi\right) \cdot \Psi^{-1}\right)_{-}^{\text {in }}\right) \Psi,
\end{aligned}
$$


where vector fields $V_{n}^{+}(\lambda), V_{n}^{-}(\lambda)$ are defined by (41) and have the coefficients polynomial respectively in $\lambda$ and $\lambda^{-1}$.

\subsection{The hierarchy and its matrix extension for symmetric system (28-29)}

The formal series in this case look symmetric with respect to the involution transposing zero and infinity [7],

$$
\begin{aligned}
& \Lambda^{\text {out }}=\ln \lambda+\varphi+\sum_{k=1}^{\infty} l_{k}^{+} \lambda^{-k}, \quad \Lambda^{\text {in }}=\ln \lambda-\varphi+\sum_{k=1}^{\infty} l_{k}^{-} \lambda^{k} \\
& M^{\text {out }}=M_{0}^{\text {out }}+\mathrm{i} \mu+\sum_{k=1}^{\infty} m_{k}^{+} \mathrm{e}^{-k \Lambda^{\text {out }}}, \quad M^{\text {in }}=M_{0}^{\text {in }}-\mathrm{i} \mu+\sum_{k=1}^{\infty} m_{k}^{-} \mathrm{e}^{k \Lambda^{\text {in }}}, \\
& M_{0}=\tau+\sum_{k=1}^{\infty} z_{k} \mathrm{e}^{k \Lambda}-\sum_{k=1}^{\infty}(-1)^{k-1} \bar{z}_{k} \mathrm{e}^{-k \Lambda},
\end{aligned}
$$

series of this form are obtained from series of the form (42) by transformation (26) with an additional twisting of a spectral variable $\lambda \rightarrow \mathrm{e}^{\varphi} \lambda, \varphi=-\frac{1}{2} \phi$. A real Euclidean reduction of an Einstein-Weyl geometry corresponds to the symmetry

$$
\begin{aligned}
& \bar{L}\left(-\bar{\lambda}^{-1}\right)=-L^{-1}(\lambda), \quad L=\mathrm{e}^{\Lambda}, \\
& \bar{M}\left(-\bar{\lambda}^{-1}\right)=M(\lambda)
\end{aligned}
$$

Generating relation (43) is unchanged, and Lax-Sato equations are modified,

$$
\begin{aligned}
& \left(\partial_{z_{n}}-\left(\frac{\lambda\{\Lambda, M\}_{\left(\lambda, z_{n}\right)}}{\{\Lambda, M\}}\right)_{+}^{\text {out }} \partial_{\tau}+\left(\frac{\{\Lambda, M\}_{\left(\tau, z_{n}\right)}}{\{\Lambda, M\}}\right)_{+}^{\text {out }} \lambda \partial_{\lambda}\right)\left(\begin{array}{c}
\Lambda \\
M
\end{array}\right)=0, \\
& \left(\partial_{\bar{z}_{n}}-\left(\frac{\lambda\{\Lambda, M\}_{\left(\lambda, \bar{z}_{n}\right)}}{\{\Lambda, M\}}\right)_{-}^{\text {in }} \partial_{\tau}+\left(\frac{\{\Lambda, M\}_{\left(\tau, \bar{z}_{n}\right)}}{\{\Lambda, M\}}\right)_{-}^{\text {in }} \lambda \partial_{\lambda}\right)\left(\begin{array}{c}
\Lambda \\
M
\end{array}\right)=0,
\end{aligned}
$$

Where the definition of projector $(\ldots)_{-}$is changed to give non-positive powers of the series, $\{f, g\}_{(x, y)}:=f_{x} g_{y}-f_{y} g_{x}$ (two-dimensional Jacobian). Lax-Sato equations (52) for $z=z_{1}, \bar{z}=\bar{z}_{1}$ correspond to Lax pair (27).

Reduction (51) can be provided using a symmetric Riemann-Hilbert problem

$$
\bar{R}\left(-\mathrm{e}^{\bar{\Lambda}^{\text {out }}}, \bar{M}^{\text {out }}\right)=R\left(\mathrm{e}^{-\Lambda^{\text {in }}}, M^{\text {in }}\right),
$$

A matrix extension is constructed similar to the preceding case. To preserve a symmetric form of the series with respect to an involution of zero and infinity (needed to perform reductions), we do not fix the normalisation and consider series of the form

$$
\Psi^{\text {out }}=\sum_{n=0}^{\infty} \Psi_{n}^{+}(\mathbf{t}) \lambda^{-n}, \quad \Psi^{\text {in }}=\sum_{n=0}^{\infty} \Psi_{n}^{-}(\mathbf{t}) \lambda^{n} .
$$


Extended Lax-Sato equations are analogous to equations (49),

$$
\begin{aligned}
& \partial_{z_{n}}\left(\begin{array}{c}
\Lambda \\
M
\end{array}\right)=V_{n}^{+}(\lambda)\left(\begin{array}{c}
\Lambda \\
M
\end{array}\right), \quad \partial_{\bar{z}_{n}}\left(\begin{array}{c}
\Lambda \\
M
\end{array}\right)=V_{n}^{-}(\lambda)\left(\begin{array}{c}
\Lambda \\
M
\end{array}\right), \\
& \left\{\partial_{z_{n}}-V_{n}^{+}(\lambda)\right\} \Psi=\left(\left(\left\{\partial_{z_{n}}-V_{n}^{+}(\lambda)\right\} \Psi\right) \cdot \Psi^{-1}\right)_{+}^{\text {out }} \Psi, \\
& \left\{\partial_{\bar{z}_{n}}-V_{n}^{-}(\lambda)\right\} \Psi=\left(\left(\left\{\partial_{\bar{z}_{n}}-V_{n}^{-}(\lambda)\right\} \Psi\right) \cdot \Psi^{-1}\right)_{-}^{\text {in }} \Psi,
\end{aligned}
$$

taking into account a modification of vector fields and projectors corresponding to equations (52). Lax-Sato equations (54) for $z=z_{1}, \bar{z}=\bar{z}_{1}$ correspond to Lax pair (31), (32).

\section{Acknowledgements}

The author is sincerely grateful to E.V. Ferapontov for useful discussions.

\section{Conflict of Interest}

The author declares no conflicts of interest.

\section{References}

[1] L.V. Bogdanov, Matrix Extension of the Manakov-Santini System and an Integrable Chiral Model on an Einstein-Weyl Background, Theor. Math. Phys., 201(3), 1701-1709 (2019)

[2] S. V. Manakov and P. M. Santini, The Cauchy problem on the plane for the dispersionless Kadomtsev-Petviashvili equation, JETP Lett. 83 (2006) $462-466$.

[3] S. V. Manakov and P. M. Santini, A hierarchy of integrable PDEs in $2+1$ dimensions associated with 2-dimensional vector fields, Theor. Math. Phys. 152 (2007) 1004-1011.

[4] M. Dunajski, E.V. Ferapontov and B. Kruglikov, On the Einstein-Weyl and conformal self-duality equations, Journal of Mathematical Physics 56(8), 083501 (2015).

[5] M. Dunajski, Solitons, Instantons, and Twistors, Oxford University Press (2010)

[6] R.S. Ward, Soliton solutions in an integrable chiral model in $2+1$ dimensions, J. Math. Phys. 29, 386389 (1988).

[7] L.V. Bogdanov, Non-Hamiltonian generalizations of the dispersionless 2DTL hierarchy, J. Phys. A: Math. Theor. 43, 434008 (2010) 
[8] E.V. Ferapontov and B.S. Kruglikov, Dispersionless integrable systems in 3D and Einstein-Weyl geometry, J. Differential Geom. Volume 97, Number 2 (2014), 215-254.

[9] R. Penrose, Nonlinear gravitons and curved twistor theory, General Relativity and Gravitation 7 (1976) 31-52.

[10] M. F. Atiyah, N. J. Hitchin and I. M. Singer, Self-duality in four dimensional Riemannian geometry, Proc. Roy. Soc. London A 362 (1978) 425461.

[11] N.J. Hitchin, Complex manifolds and Einstein's equations, Twistor geometry and nonlinear systems (Primorsko, 1980), 73-99, Lecture Notes in Math. 970, Springer, Berlin-New York (1982).

[12] David M.J. Calderbank, Integrable Background Geometries, SIGMA 10 (2014), 034.

[13] L.V. Bogdanov, SDYM equations on the self-dual background, J. Phys. A 50, 19LT02 (2017)

[14] H. Pedersen and K.P. Tod, Three Dimensional Einstein-Weyl Geometry, Advances in Mathematics, 97 (1), 74-109 (1993)

[15] L.V. Bogdanov, DunajskiTod equation and reductions of the generalized dispersionless 2DTL hierarchy, Phys. Lett. A 376(45), 28942898 (2012)

[16] S.V. Manakov and V.E. Zakharov, Three-dimensional model of relativisticinvariant field theory, integrable by the inverse scattering transform, Lett. Math. Phys. 5, 247253 (1981) 